\title{
Vertex correction and Ward identity in the U(1) gauge theory with Fermi surface
}

\author{
Ki-Seok $\operatorname{Kim}^{1,2}$ \\ ${ }^{1}$ Asia Pacific Center for Theoretical Physics, Hogil Kim Memorial building 5th floor, \\ POSTECH, Hyoja-dong, Namgu, Pohang 790-784, Korea \\ ${ }^{2}$ Department of Physics, Pohang University of Science and Technology, Pohang, Gyeongbuk 790-784, Korea
}

(Dated: October 4, 2018)

\begin{abstract}
We show that introduction of vertex corrections in the fully self-consistent ladder approximation does not modify dynamics of spinons and gauge fluctuations in the U(1) gauge theory with Fermi surface.

PACS numbers: 71.10.-w, 71.10.Hf, 71.27.+a

\section{INTRODUCTION}

\section{BEYOND THE ELIASHBERG FRAMEWORK}

Spin liquid has been a cornerstone in the gauge theory approach for strongly correlated electrons ${ }^{1}$ as Fermi liquid in the Landau-Ginzburg-Wilson framework for phase transitions ${ }^{2}$. An effective field theory is often given by compact $\mathrm{U}(1)$ gauge theory ${ }^{\underline{3}}$, implying that "magnetic monopole" excitations should be irrelevant in order to be self-consistent for the theory. Two kinds of mechanisms have been proposed, resulting from either spinon dynamics with Fermi surface ${ }^{4}-\underline{\underline{7}}$ or existence of a topological term ${ }^{8}$ associated with anomaly in the Dirac theory ${ }^{9,10}$. After deconfinement is demonstrated, an important task is to solve the non-compact $\mathrm{U}(1)$ gauge theory.

A standard technique is the large- $N$ approximation, where the spin degeneracy of a spinon is extended from $\sigma=\uparrow, \downarrow$ to $\sigma=1, \ldots, N$. The $N \rightarrow \infty$ limit was believed to suppress higher-order quantum loop-corrections in the Fermi surface problem 11 just as the case of the relativis-

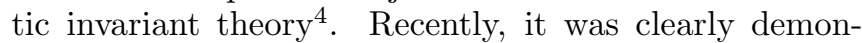
strated that the Fermi surface problem is still strongly interacting even in the large- $N$ limit, meaning that all planar Feynmann diagrams should be summed as the non-abelian gauge theory with Lorentz invariance $\frac{12}{2}$.

This observation suggests that dynamics of fermions (spinons) and gauge fluctuations can be modified by more-loops quantum corrections, that is, the exponent in the frequency dependence of the spinon self-energy may have a nontrivial correction, affecting transport properties of spinons. Actually, the lowest-order vertex correction associated with the Aslamasov-Larkin diagram is shown to cause such a correction proportional to $1 / N$ although the lowest-order vertex correction associated with the ladder diagram does not change the dynamics of both fermions and collective bosons $\frac{13}{}$.

In this paper we perform an infinite-order summation for the ladder-type vertex correction and find no anomalous correction for the exponent in the frequency dependence of the fermion self-energy. In other words, dynamics of both spinons and gauge bosons remains the same as the case without vertex corrections $\frac{14}{4}$. We prove this result based on the Ward identity $\stackrel{15}{ }$, asymptotically exact in the low energy limit.

\section{A. Review on the Eliashberg theory}

We start from an effective U(1) gauge theory with one patch in one time and two space dimensions $\frac{12}{2}$

$$
\mathcal{L}=f_{\sigma}^{\dagger}\left(\eta \partial_{\tau}-i \partial_{x}-\partial_{y}^{2}\right) f_{\sigma}+\frac{e}{\sqrt{N}} a f_{\sigma}^{\dagger} f_{\sigma}+a\left(-\partial_{y}^{2}\right)^{\frac{z-1}{2}} a
$$

where $f_{\sigma}$ and $a$ represent fermionic spinons and $\mathrm{U}(1)$ gauge fluctuations, respectively, emerging in the $\mathrm{U}(1)$ spin liquid state ${ }^{\underline{1}}$. $e$ is an internal gauge charge of a spinon and $N$ is the spin degeneracy. $\eta$ is an infinitesimal coefficient to control artificial divergences in quantum corrections, which can be cured by self-energy corrections $\frac{12}{2} . z$ is the dynamical exponent determining the dispersion relation of gauge fluctuations. It is given by $z=3$ for several problems such as ferromagnetic or nematic quantum criticality including the present spin liquid problem 11 while $z=2$ in the spin density wave ordering 16 . Both the Fermi velocity $v_{F}$ and the curvature $1 / m$ are set to one.

It was shown that either scattering with small momentum transfer or that with $2 k_{F}$ (twice of the Fermi momentum) is relevant in the Fermi surface problem 2,5 . In the one patch approximation 12 only forward scattering, identified with $g_{4}$ in the $g$-ology of the one dimensional problem 17 , is taken into account while another forward scattering $\left(g_{2}\right)$ and back scattering $\left(g_{1}\right)$ are neglected. Such scattering channels can be introduced in the two patch approximation $\underline{13}$.

The previous large- $N$ analysis without vertex corrections coincides with the Eliashberg approximation $\frac{11}{1}$, in- 
troducing only self-energy corrections

$$
\begin{aligned}
\Pi\left(q_{0}, q\right) & =\frac{e^{2}}{N} \int \frac{d k_{0}}{2 \pi} \int \frac{d^{2} k}{(2 \pi)^{2}} G_{\sigma}\left(k_{0}+q_{0}, k+q\right) G_{\sigma}\left(k_{0}, k\right) \\
& =\gamma_{b} \frac{\left|q_{0}\right|}{\left|q_{y}\right|} \\
\Sigma\left(k_{0}\right) & =-\frac{e^{2}}{N} \int \frac{d q_{0}}{2 \pi} \int \frac{d^{2} q}{(2 \pi)^{2}} G_{\sigma}\left(k_{0}+q_{0}, k+q\right) D\left(q_{0}, q\right) \\
& =-i \frac{\lambda_{b}}{N} \operatorname{sgn}\left(k_{0}\right)\left|k_{0}\right|^{2 / z}
\end{aligned}
$$

where the spinon Green's function and gauge propagator are given by

$$
\begin{aligned}
& G_{\sigma}\left(k_{0}, k\right)=\frac{1}{i \eta k_{0}+k_{x}+k_{y}^{2}-\Sigma\left(k_{0}\right)}, \\
& D\left(q_{0}, q\right)=\frac{1}{\left|q_{y}\right|^{z-1}+\Pi\left(q_{0}, q\right)},
\end{aligned}
$$

respectively. The main point is that dynamics of gauge fluctuations is given by the Landau damping term with the damping coefficient $\gamma_{b}$ proportional to $k_{F}^{-1}$, resulting from Fermi-surface fluctuations, while spinon dynamics shows non-Fermi liquid dependence in frequency of its self-energy, given by $2 / z$ with a constant $\lambda_{b}$.

The problem to address in this paper is whether the anomalous exponent $2 / z$ will be modified or not when vertex corrections are taken into account in a nonperturbative way, i.e., up to an infinite order. It turns out that the gauge dynamics cannot be modified from the Landau damping dynamics in the one patch approximation while the fermion dynamics is expected to have some corrections $\frac{12}{2}$. Even in the two patch approximation, the gauge dynamics is still unchanged while fermions were shown to have $1 / N$ modification for the frequency exponent in the perturbative approach based on the Eliashberg solution 13 .

\section{B. Self-consistent ladder approximation}

We introduce vertex corrections in a completely nonperturbative way based on the ladder approximation. Then, we obtain full self-consistent equations

$$
\begin{aligned}
& \Pi\left(q_{0}, q\right)=\frac{e^{2}}{N} \int \frac{d k_{0}}{2 \pi} \int \frac{d^{2} k}{(2 \pi)^{2}} \Lambda\left(k_{0}+q_{0}, k+q ; k_{0}, k\right) \\
& G_{\sigma}\left(k_{0}+q_{0}, k+q\right) G_{\sigma}\left(k_{0}, k\right), \\
& \Sigma\left(k_{0}\right)=-\frac{e^{2}}{N} \int \frac{d q_{0}}{2 \pi} \int \frac{d^{2} q}{(2 \pi)^{2}} \Lambda\left(k_{0}+q_{0}, k+q ; k_{0}, k\right) \\
& G_{\sigma}\left(k_{0}+q_{0}, k+q\right) D\left(q_{0}, q\right),
\end{aligned}
$$

where $\Lambda\left(k_{0}+q_{0}, k+q ; k_{0}, k\right)$ is the vertex function given by

$$
\begin{aligned}
& \Lambda\left(k_{0}+q_{0}, k+q ; k_{0}, k\right)=1-\frac{e^{2}}{N} \int \frac{d l_{0}}{2 \pi} \int \frac{d^{2} l}{(2 \pi)^{2}} \\
& \Lambda\left(k_{0}+q_{0}-l_{0}, k+q-l ; k_{0}-l_{0}, k-l\right) D\left(l_{0}, l\right) \\
& G_{\sigma}\left(k_{0}+q_{0}-l_{0}, k+q-l\right) G_{\sigma}\left(k_{0}-l_{0}, k-l\right)
\end{aligned}
$$
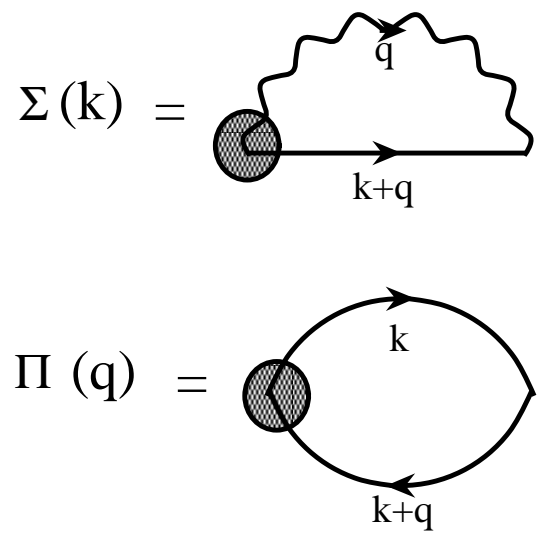

FIG. 1: Fermion self-energy $\Sigma(k)$ and boson self-energy $\Pi(q)$, where the thick line represents the fermion Green's function and the wavy line does the gauge propagator. The shaded region can be any renormalized vertex.

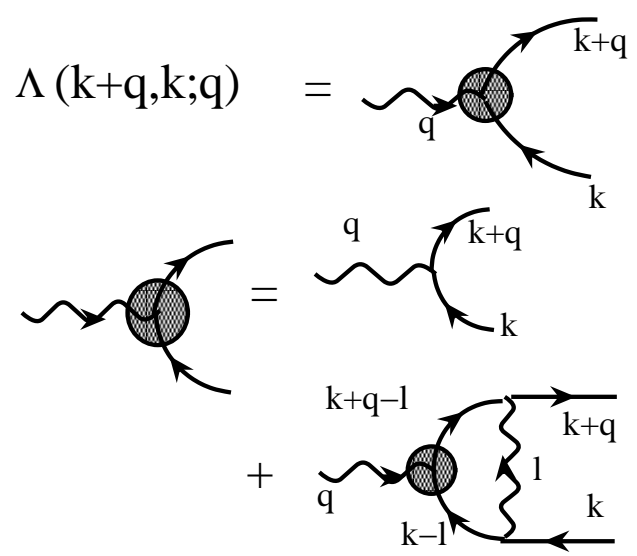

FIG. 2: (Color online) The ladder vertex correction turns out to be irrelevant in the Eliashberg solution.

in the ladder approximation. Fig. 1 represents Eq. (4) and Fig. 2 displays Eq. (5).

In order to solve these three coupled integral equations, we consider the Ward identity $\underline{17}$

$$
\begin{aligned}
& \left.i \eta q_{0}\right\urcorner\left(k_{0}+q_{0}, k+q ; k_{0}, k\right)+q_{x} \Lambda\left(k_{0}+q_{0}, k+q ; k_{0}, k\right) \\
& +q_{y} \digamma\left(k_{0}+q_{0}, k+q ; k_{0}, k\right) \\
& =G_{\sigma}^{-1}\left(k_{0}+q_{0}, k+q\right)-G_{\sigma}^{-1}\left(k_{0}, k\right),
\end{aligned}
$$

where $\neg\left(k_{0}+q_{0}, k+q ; k_{0}, k\right)$ is the scalar vertex while $\Lambda\left(k_{0}+q_{0}, k+q ; k_{0}, k\right)$ and $\digamma\left(k_{0}+q_{0}, k+q ; k_{0}, k\right)$ are vector vertices. There is a special relation between the scalar vertex and vector one in one dimension due to the kinematic constraint, that is, the vector vertex is proportional to the scalar vertex with the Fermi velocity $\underline{\underline{17}}$. This is the reason why the one dimensional problem is exactly solvable. On the other hand, such a relation does not exist above one dimension, thus it is necessary to propose an ansatz for such a relation. 
We suggests the following relation

$\begin{aligned} \neg\left(k_{0}+q_{0}, k+q ; k_{0}, k\right) & \rightarrow \Lambda\left(k_{0}+q_{0}, k+q ; k_{0}, k\right), \\ \digamma\left(k_{0}+q_{0}, k+q ; k_{0}, k\right) & \rightarrow\left(2 k_{y}+q_{y}\right) \Lambda\left(k_{0}+q_{0}, k+q ; k_{0}, k\right),\end{aligned}$

The first part denoted by $\Sigma_{1}\left(k_{0}\right)$ turns out to vanish

$$
\Sigma_{1}\left(k_{0}\right)=\frac{i e^{2}}{2 N} \int \frac{d q_{0}}{2 \pi} \int \frac{d q_{y}}{2 \pi} \frac{\operatorname{sgn}\left(q_{0}\right)}{\gamma \frac{\left|q_{0}\right|}{\left|q_{y}\right|}+\left|q_{y}\right|^{z-1}}=0 .
$$

where the first ansatz is the application of the one dimensional result while the second one is our main assumption. As a result, the vertex function is

$$
\Lambda\left(k_{0}+q_{0}, k+q ; k_{0}, k\right)=\frac{G_{\sigma}^{-1}\left(k_{0}+q_{0}, k+q\right)-G_{\sigma}^{-1}\left(k_{0}, k\right)}{g_{\sigma}^{-1}\left(k_{0}+q_{0}, k+q\right)-g_{\sigma}^{-1}\left(k_{0}, k\right)},
$$

On the other hand, the second part recovers exactly the same expression as Eq. (2) in the low energy limit

$$
\Sigma_{2}\left(k_{0}\right)=\Sigma\left(k_{0}\right)=-i \frac{\lambda}{N} \operatorname{sgn}\left(k_{0}\right)\left|k_{0}\right|^{2 / z},
$$

where $g_{\sigma}^{-1}\left(k_{0}, k\right)=i \eta k_{0}+k_{x}+k_{y}^{2}$ is a non-interacting Green's function. We note that this expression recovers both non-interacting and one dimensional cases. In appendix A we show that this ansatz is self-consistent for the vertex equation [Eq. (5)] in the low energy limit.

It is educational to check that if we apply the wellknown one dimensional ansatz for the vertex function, i.e., neglecting $\digamma\left(k_{0}+q_{0}, k+q ; k_{0}, k\right)$, given by

$$
\Lambda\left(k_{0}+q_{0}, k+q ; k_{0}, k\right) \approx \frac{G_{\sigma}^{-1}\left(k_{0}+q_{0}, k+q\right)-G_{\sigma}^{-1}\left(k_{0}, k\right)}{i \eta q_{0}+q_{x}}
$$

we find that the Landau damping dynamics for gauge fluctuations is not reproduced as follows

$$
\begin{aligned}
& \Pi\left(q_{0}, q\right)=\frac{e^{2}}{N} \frac{1}{i q_{0}+q_{x}} \int \frac{d k_{0}}{2 \pi} \int \frac{d^{2} k}{(2 \pi)^{2}}\left(G_{\sigma}\left(k_{0}, k\right)\right. \\
& \left.-G_{\sigma}\left(k_{0}+q_{0}, k+q\right)\right) \propto e^{2} \frac{i\left|q_{0}\right|}{i \eta q_{0}+q_{x}} .
\end{aligned}
$$

This implies that the one dimensional Ward identity cannot be applied to higher dimensional cases.

Inserting the vertex function Eq. (8) into the equation for the polarization function in Eq. (4), we find that the Landau damping dynamics does not change as follows

$$
\begin{aligned}
& \Pi\left(q_{0}, q\right) \\
& =\frac{e^{2}}{N} \int \frac{d k_{0}}{2 \pi} \int \frac{d^{2} k}{(2 \pi)^{2}} \frac{G_{\sigma}\left(k_{0}, k\right)-G_{\sigma}\left(k_{0}+q_{0}, k+q\right)}{i \eta q_{0}+q_{x}+2 k_{y} q_{y}+q_{y}^{2}} \\
& =\frac{i e^{2}}{2} \int \frac{d k_{0}}{2 \pi} \int \frac{d k_{y}}{2 \pi} \frac{\operatorname{sgn}\left(k_{0}+q_{0}\right)-\operatorname{sgn}\left(k_{0}\right)}{i \eta q_{0}+q_{x}+2 k_{y} q_{y}+q_{y}^{2}} \\
& =\frac{e^{2}}{8} \int \frac{d k_{0}}{2 \pi} \frac{\operatorname{sgn}\left(q_{0}\right)\left[\operatorname{sgn}\left(k_{0}+q_{0}\right)-\operatorname{sgn}\left(k_{0}\right)\right]}{\left|q_{y}\right|} \\
& =\gamma \frac{\left|q_{0}\right|}{\left|q_{y}\right|},
\end{aligned}
$$

where $\gamma$ is a modified damping coefficient.

Inserting both the vertex function [Eq. (8)] and boson self-energy [Eq. (9)] into the equation for the fermion self-energy in Eq. (4), we obtain two sectors

$$
\begin{aligned}
& \Sigma\left(k_{0}\right)=-\frac{e^{2}}{N} \int \frac{d q_{0}}{2 \pi} \int \frac{d^{2} q}{(2 \pi)^{2}} G_{\sigma}\left(k_{0}+q_{0}, k+q\right) D\left(q_{0}, q\right) \\
& \frac{G_{\sigma}^{-1}\left(k_{0}+q_{0}, k+q\right)-G_{\sigma}^{-1}\left(k_{0}, k\right)}{i \eta q_{0}+q_{x}+2 k_{y} q_{y}+q_{y}^{2}} \\
& \equiv \Sigma_{1}\left(k_{0}\right)+\Sigma_{2}\left(k_{0}\right) .
\end{aligned}
$$

where $\lambda$ is a modified constant. We show the derivation of this result in appendix B.

\section{Discussion}

An essential point is that the ladder-vertex equation [Eq. (5)] is solved, resorting to the Ward identity, where the ansatz for the relationship between the scalar and vector vertices [Eq. (7)] is introduced to result in the relationship between the vertex function and fermion Green's function [Eq. (8)]. Justification for Eq. (8) lies in the fact that the boson self-energy should be given by the Landau damping solution. In the one patch formulation higher order quantum corrections are shown to vanish identically because all poles in the integral expression are in the same half plane, implying that the Landau damping solution is exact $\frac{12}{2}$. Considering the structure of the boson self-energy with the ladder-vertex correction [Eq. (9)], Eq. (8) seems to be generic. In addition, Eq. (8) recovers not only the non-interacting case but also the one dimensional physics completely. The relationship between Eq. (7) and Eq. (8) is unique as far as the $\mathrm{y}$-current vertex is linearly related with the $\mathrm{x}$-current or scalar vertex.

Can we use the Ward identity in this approximation scheme? Usually speaking, the Ward identity is on the relationship between full vertex corrections and corresponding Green's functions. Actually, the Ward identity and the special relation between the vector and scalar vertices in one dimension are satisfied for the fully renormalized vertex. In fact, the Ward identity should be always satisfied in any approximation scheme because it guarantees conservation of the system. In this sense the Ward identity may be regarded as another phrase of the conserving approximation. Mathematically speaking, the conserving approximation can be derived from the Luttinger-Ward functional approach, where fully self-consistent sets of equations derived from the Luttinger-Ward functional respect the Ward identity automatically $\underline{18,19}$. Of course, this is not exact. A good example can be found in the impurity problem, called the conserving self-consistent t-matrix approximation ${ }^{20}$.

Another important assumption is that singular dependence of the fermion self-energy occurs from frequency instead of momentum. Though this is a common result 
within the Eliashberg approximation ${ }^{11}$, there is no reason a priori, for this assumption to remain valid as soon as ladder-type vertex corrections are included. In particular, the self-consistent calculations performed in the appendices are carried out within this assumption, which greatly simplifies the computations. It was argued that the fermion self-energy has the same frequency dependence as the Eliashberg solution and there is no singular momentum dependence in the perturbative evaluation of the one patch formulation up to an infinite order based on the Eliashberg solution ${ }^{12,14}$, implying no anomalous exponent for the fermion Green's function, although one can not remove the possibility that the summation for coefficients from higher order quantum corrections may be singular. In addition, ladder-type vertex corrections turn out not to change the Elaishberg solution in the perturbative calculation of the two patch formulation up to the lowest order ${ }^{13}$. In this respect our result is not surprising but expected from the perturbative analysis in both one-patch ${ }^{12}$ and two-patch ${ }^{13}$ formulations.

However, special types of quantum corrections involved with $2 k_{F}$ momentum transfer were shown to cause the singular momentum dependence for the fermion selfenergy in the two patch formulation, giving rise to an anomalous exponent for the fermion Green's function ${ }^{13}$. Unfortunately, these quantum processes are not introduced in the ladder approximation, given by the Aslamasov-Larkin diagrams. This leads us to consider the Aslamasov-Larkin vertex up to an infinite order, shown in Fig. 3. Frankly speaking, this consideration is not completely new, already investigated in the context of the superconducting instability although it corresponds to the particle-particle channe ${ }^{21}$. An interesting point is that such vertices are singulary enhanced in the $U(1)$ spin liquid state, causing anomalous critical exponents according to the perturbative evaluation. There is another Aslamasov-Larkin vertex correction in the particle-particle channel associated with superconductivity, competing with the $2 k_{F}$ particle-hole instability. One problem in this consideration is to construct the self-consistent conserving approximation, not addressed clearly as far as we know. When this self-consistent conserving framework is settled, we can check whether the vertex function [Eq. (8)] from the Ward identity allows the self-consistent solution with new critical exponents or not. If it works, we have a powerful framework.

This discussion reminds us of the conserving selfconsistent t-matrix approximation (CTMA) for the single impurity problem ${ }^{20}$, introduced to overcome the failure of the non-crossing approximation (NCA) in the exactly screened case, i.e., the absence of the strong coupling fixed point below the Kondo temperature. Here, the NCA is analogous to the Eliashberg approximation while the CTMA is parallel with the self-consistent $2 k_{F}$ treatment. Although the CTMA does not change the critical exponents of the NCA in the over-screened case, it cures several problems associated with thermodynamics 20 . In the present situation the self-consistent $2 k_{F}$ treatment

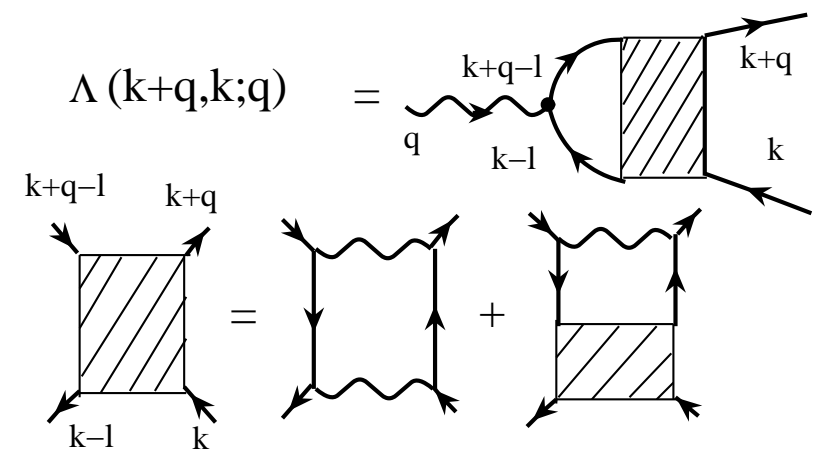

FIG. 3: (Color online) The Aslamasov-Larkin vertex correction in the particle-hole channel, where $2 k_{F}$ momentum transfer gives rise to an anomalous critical exponent for the fermion propagator. This situation is quite analogous to the superconducting instability. Actually, there is another AslamasovLarkin vertex correction in the particle-particle channel, competing with the $2 k_{F}$ particle-hole instability. Inserting these Aslamasov-Larkin vertex corrections into the shaded regions of Fig. 1, one can construct self-consistent equations for fermion and boson self-energies.

may change the critical exponents of the Eliashberg approximation. It will be really interesting to investigate this type of diagrams near future.

\section{SUMMARY}

In this study we solved three coupled integral equations for boson and fermion self-energies with vertex corrections. The key point is to make an ansatz for the vertex function in terms of the fermion Green's function based on the Ward identity. Resorting to this ansatz, we find a set of full self-consistent solutions in the ladder approximation for the vertex correction, where both boson and fermion self-energy corrections do not have any modification, compared with the Eliashberg approximation. This implies that if the anomalous exponent arises in the fermion self-energy, it may result from the class of Aslamasov-Larkin diagrams, not taken into account in our study. This remains as an important future work.

K.-S. Kim appreciate an inspiring lecture of J. McGreevy in the string spring school in ICTP (2010), motivating us to investigate the present problem. K.-S. Kim was supported by the National Research Foundation of Korea (NRF) grant funded by the Korea government (MEST) (No. 2010-0074542).

Appendix A: Self-consistency between the Ward identity and the ladder approximation for the vertex function in the low energy limit

In appendix $\mathrm{A}$ we show that the ladder vertex in Eq. (5) satisfies the Ward identity with the ansatz Eq. (8). 
In other words, the ansatz Eq. (8) is shown to be selfconsistent at least in the ladder approximation.

Inserting Eq. (8) into the right hand side (R.H.S) in Eq. (5), we obtain

$$
\begin{aligned}
& \text { R.H.S. }-1=-\frac{e^{2}}{N} \int \frac{d l_{0}}{2 \pi} \int \frac{d^{2} l}{(2 \pi)^{2}} D\left(l_{0}, l\right) \\
& \frac{G_{\sigma}\left(k_{0}-l_{0}, k-l\right)-G_{\sigma}\left(k_{0}+q_{0}-l_{0}, k+q-l\right)}{g_{\sigma}^{-1}\left(k_{0}+q_{0}-l_{0}, k+q-l\right)-g_{\sigma}^{-1}\left(k_{0}-l_{0}, k-l\right)} .
\end{aligned}
$$

Performing the $l_{x}$ integration, we obtain

$$
\begin{aligned}
& \text { R.H.S. }-1=\frac{i e^{2}}{2 N} \int_{-\infty}^{\infty} \frac{d l_{0}}{2 \pi} \int_{-\infty}^{\infty} \frac{d l_{y}}{2 \pi} \frac{1}{\gamma \frac{\left|l_{0}\right|}{\left|l_{y}\right|}+\left|l_{y}\right|^{z-1}} \\
& \frac{\operatorname{sgn}\left(k_{0}-l_{0}\right)-\operatorname{sgn}\left(k_{0}+q_{0}-l_{0}\right)}{i \eta q_{0}+q_{x}+2\left(k_{y}-l_{y}\right) q_{y}+q_{y}^{2}}
\end{aligned}
$$

Integrating over the frequency $l_{0}$, we obtain

$$
\begin{aligned}
& \text { R.H.S. }-1=\frac{i e^{2}}{4 \pi \gamma N} \int_{0}^{\infty} \frac{d l_{y}}{2 \pi} \frac{1}{2 q_{y}}\left\{\ln \left(\gamma\left|k_{0}+q_{0}\right|+l_{y}^{z}\right)\right. \\
& \left.-\ln \left(\gamma\left|k_{0}\right|+l_{y}^{z}\right)\right\}\left(\frac{-i \eta q_{0}-q_{x}-2 k_{y} q_{y}-q_{y}^{2}}{i \eta q_{0}+q_{x}+2\left(k_{y}+l_{y}\right) q_{y}+q_{y}^{2}}\right. \\
& \left.+\frac{i \eta q_{0}+q_{x}+2 k_{y} q_{y}+q_{y}^{2}}{i \eta q_{0}+q_{x}+2\left(k_{y}-l_{y}\right) q_{y}+q_{y}^{2}}\right) \equiv \mathbf{A}+\mathbf{B} .
\end{aligned}
$$

The first ln contribution can be approximated as follows

$$
\begin{aligned}
& \mathbf{A} \approx \frac{i e^{2}}{4 \pi \gamma N} \int_{0}^{\left(\gamma\left|k_{0}+q_{0}\right|\right)^{1 / z}} \frac{d l_{y}}{2 \pi} \frac{1}{2 q_{y}} \\
& \left\{\ln \left(\gamma\left|k_{0}+q_{0}\right|\right)\right\}\left(\frac{-i \eta q_{0}-q_{x}-2 k_{y} q_{y}-q_{y}^{2}}{i \eta q_{0}+q_{x}+2\left(k_{y}+l_{y}\right) q_{y}+q_{y}^{2}}\right. \\
& \left.+\frac{i \eta q_{0}+q_{x}+2 k_{y} q_{y}+q_{y}^{2}}{i \eta q_{0}+q_{x}+2\left(k_{y}-l_{y}\right) q_{y}+q_{y}^{2}}\right) \\
& +\frac{i e^{2}}{4 \pi \gamma N} \int_{\left(\gamma\left|k_{0}+q_{0}\right|\right)^{1 / z}}^{\Lambda} \frac{d l_{y}}{2 \pi} \frac{1}{2 q_{y}} \\
& \left\{\ln l_{y}^{z}\right\}\left(\frac{-i \eta q_{0}-q_{x}-2 k_{y} q_{y}-q_{y}^{2}}{i \eta q_{0}+q_{x}+2\left(k_{y}+l_{y}\right) q_{y}+q_{y}^{2}}\right. \\
& \left.+\frac{i \eta q_{0}+q_{x}+2 k_{y} q_{y}+q_{y}^{2}}{i \eta q_{0}+q_{x}+2\left(k_{y}-l_{y}\right) q_{y}+q_{y}^{2}}\right) .
\end{aligned}
$$

Expanding the first sector in $l_{y}$, we obtain

$$
\begin{aligned}
& \mathbf{A} \approx \frac{i e^{2}}{4 \pi \gamma N} \int_{0}^{\left(\gamma\left|k_{0}+q_{0}\right|\right)^{1 / z}} \frac{d l_{y}}{2 \pi} \\
& \left\{\ln \left(\gamma\left|k_{0}+q_{0}\right|\right)\right\} \frac{2 l_{y}}{i \eta q_{0}+q_{x}+2 k_{y} q_{y}+q_{y}^{2}} \\
& +\frac{i e^{2}}{4 \pi \gamma N} \int_{\left(\gamma\left|k_{0}+q_{0}\right|\right)^{1 / z}}^{\Lambda} \frac{d l_{y}}{2 \pi} \frac{1}{2 q_{y}} \\
& \left\{\ln l_{y}^{z}\right\}\left(\frac{-i \eta q_{0}-q_{x}-2 k_{y} q_{y}-q_{y}^{2}}{i \eta q_{0}+q_{x}+2\left(k_{y}+l_{y}\right) q_{y}+q_{y}^{2}}\right. \\
& \left.+\frac{i \eta q_{0}+q_{x}+2 k_{y} q_{y}+q_{y}^{2}}{i \eta q_{0}+q_{x}+2\left(k_{y}-l_{y}\right) q_{y}+q_{y}^{2}}\right) .
\end{aligned}
$$

One will realize that the first term is associated with the self-energy in the Eliashberg approximation. Evaluating the $\mathbf{B}$ term in the same way as $\mathbf{A}$ and gathering both $\mathbf{A}$ and $\mathbf{B}$, we reach the final expression

$$
\begin{aligned}
& \Lambda\left(k_{0}+q_{0}, k+q ; k_{0}, k\right)-1=\mathbf{A}+\mathbf{B} \\
& =-\frac{\Sigma\left(k_{0}+q_{0}\right)-\Sigma\left(k_{0}\right)}{g_{\sigma}^{-1}\left(k_{0}+q_{0}, k+q\right)-g_{\sigma}^{-1}\left(k_{0}, k\right)} \\
& +\mathcal{F}\left(k_{0}+q_{0}, k+q ; k_{0}, k\right),
\end{aligned}
$$

where

$$
\begin{aligned}
& \mathcal{F}\left(k_{0}+q_{0}, k+q ; k_{0}, k\right) \equiv \frac{i e^{2}}{4 \pi \gamma N} \int_{\left(\gamma\left|k_{0}+q_{0}\right|\right)^{1 / z}}^{\Lambda} \frac{d l_{y}}{2 \pi} \frac{1}{2 q_{y}} \\
& \left\{\ln l_{y}^{z}\right\}\left(\frac{-i \eta q_{0}-q_{x}-2 k_{y} q_{y}-q_{y}^{2}}{i \eta q_{0}+q_{x}+2\left(k_{y}+l_{y}\right) q_{y}+q_{y}^{2}}\right. \\
& \left.+\frac{i \eta q_{0}+q_{x}+2 k_{y} q_{y}+q_{y}^{2}}{i \eta q_{0}+q_{x}+2\left(k_{y}-l_{y}\right) q_{y}+q_{y}^{2}}\right) \\
& -\frac{i e^{2}}{4 \pi \gamma N} \int_{\left(\gamma\left|k_{0}\right|\right)^{1 / z}}^{\Lambda} \frac{d l_{y}}{2 \pi} \frac{1}{2 q_{y}} \\
& \left\{\ln l_{y}^{z}\right\}\left(\frac{-i \eta q_{0}-q_{x}-2 k_{y} q_{y}-q_{y}^{2}}{i \eta q_{0}+q_{x}+2\left(k_{y}+l_{y}\right) q_{y}+q_{y}^{2}}\right. \\
& \left.+\frac{i \eta q_{0}+q_{x}+2 k_{y} q_{y}+q_{y}^{2}}{i \eta q_{0}+q_{x}+2\left(k_{y}-l_{y}\right) q_{y}+q_{y}^{2}}\right) .
\end{aligned}
$$

It is not difficult to observe that $\mathcal{F}\left(k_{0}+q_{0}, k+q ; k_{0}, k\right)$ is irrelevant in the low energy limit due to the frequency and momentum dependence in the numerator, giving rise to higher order corrections to the fermion self-energy. We conclude that Eq. (8) is asymptotically correct in the low energy limit. 


\section{Appendix B: Evaluation of $\Sigma_{2}\left(k_{0}\right)$}

In appendix $\mathrm{B}$ we evaluate $\Sigma_{2}\left(k_{0}\right)$. Performing momentum and frequency integrals, we obtain

$$
\begin{aligned}
& \Sigma_{2}\left(k_{0}\right)=\frac{e^{2}}{N} \int \frac{d q_{0}}{2 \pi} \int \frac{d^{2} q}{(2 \pi)^{2}} \frac{1}{i \eta q_{0}+q_{x}+2 k_{y} q_{y}+q_{y}^{2}} \\
& \frac{i \eta k_{0}+k_{x}+k_{y}^{2}-\Sigma\left(k_{0}\right)}{i \eta\left(k_{0}+q_{0}\right)+\left(k_{x}+q_{x}\right)+\left(k_{y}+q_{y}\right)^{2}-\Sigma\left(k_{0}+q_{0}\right)} \\
& \frac{1}{\gamma \frac{\left|q_{0}\right|}{\left|q_{y}\right|}+\left|q_{y}\right| z-1} \\
& =\frac{e^{2}}{N} \int \frac{d q_{0}}{2 \pi} \int \frac{d q_{y}}{2 \pi} \int \frac{d q_{x}}{2 \pi} \frac{1}{\gamma \frac{\left|q_{0}\right|}{\left|q_{y}\right|}+\left|q_{y}\right|^{z-1}} \\
& \frac{i \eta k_{0}+k_{x}+k_{y}^{2}-\Sigma\left(k_{0}\right)}{i \eta k_{0}+k_{x}+k_{y}^{2}-\Sigma\left(k_{0}+q_{0}\right)}\left\{\frac{1}{\left(i \eta q_{0}+q_{x}+2 k_{y} q_{y}+q_{y}^{2}\right)}\right. \\
& \left.-\frac{i \eta\left(k_{0}+q_{0}\right)+\left(k_{x}+q_{x}\right)+\left(k_{y}+q_{y}\right)^{2}-\Sigma\left(k_{0}+q_{0}\right)}{i n}\right\} \\
& =-\frac{i e^{2}}{2 \pi \gamma^{1-2 / z}}\left\{\int_{0}^{\Lambda} d y \frac{y}{1+y^{z}}\right\} \\
& \int \frac{d q_{0}}{2 \pi} \frac{i \eta k_{0}+k_{x}+k_{y}^{2}-\Sigma\left(k_{0}\right)}{i \eta k_{0}+k_{x}+k_{y}^{2}-\Sigma\left(k_{0}+q_{0}\right)} \\
& \frac{\operatorname{sgn}\left(q_{0}\right)-\operatorname{sgn}\left(k_{0}+q_{0}\right)}{q_{0}^{1-2 / z}} \approx-i \frac{\lambda}{N} \operatorname{sgn}\left(k_{0}\right)\left|k_{0}\right|^{2 / z} .
\end{aligned}
$$

The vertex correction does not change the scaling for frequency in the ladder approximation.
1 P. A. Lee, N. Nagaosa, and X.-G. Wen, Rev. Mod. Phys. 78, 17 (2006).

2 R. Shankar, Rev. Mod. Phys. 66, 129 (1994).

3 A. M. Polyakov, Gauge Fields and Strings (Harwood Academic Publishers, 1987).

4 M. Hermele, T. Senthil, M. P. A. Fisher, P. A. Lee, N. Nagaosa, and X.-G. Wen, Phys. Rev. B 70, 214437 (2004); Since this study considers the $\mathrm{U}(1)$ gauge theory with $N$ flavor massless Dirac fermions, it differs from the Fermi surface problem, precisely speaking. However, if only the confinement issue is taken into account, mechanism for irrelevance of magnetic monopoles seems to be basically same between the Dirac-fermion and Fermi surface problems in respect that matters screen such gauge interactions.

5 Sung-Sik Lee, Phys. Rev. B 78, 085129 (2008).

6 N. Nagaosa, Phys. Rev. Lett. 71, 4210 (1993).

7 Ki-Seok Kim, Phys. Rev. B 72, 245106 (2005).

8 T. Senthil, A. Vishwanath, L. Balents, S. Sachdev, and M. P. A. Fisher, Science 303, 1490 (2004); T. Senthil, L. Balents, S. Sachdev, A. Vishwanath, and M. P.A. Fisher, Phys. Rev. B 70, 144407 (2004).

9 A. Tanaka and X. Hu, Phys. Rev. Lett. 95, 036402 (2005); A. Tanaka and X. Hu, Phys. Rev. Lett. 88, 127004 (2002).

10 Ki-Seok Kim; Phys. Rev. B 78, 195113 (2008); Ki-Seok Kim, Phys. Rev. B 72, 214401 (2005).

11 J. Rech, C. Pepin, and A. V. Chubukov, Phys. Rev. B 74, 195126 (2006).

12 Sung-Sik Lee, Phys. Rev. B 80, 165102 (2009).

13 Max A. Metlitski and S. Sachdev, arXiv:1001.1153 (un- published).

14 David F. Mross, J. McGreevy, H. Liu, and T. Senthil, arXiv:1003.0894 (unpublished); In this study the authors introduce a new expansion parameter $\epsilon=z-2$ following the previous paper [J. Polchinski, Nucl. Phys. B 422, 617 (1994)], and find the large- $N$ expansion controllable in the $\epsilon \rightarrow 0$ limit, recovering the result of the Eliashberg approximation.

15 A. V. Chubukov, Phys. Rev. B 72, 085113 (2005).

16 T. Moriya and J. Kawabata, J. Phys. Soc. Jpn. 34, 639 (1973); T. Moriya and J. Kawabata, J. Phys. Soc. Jpn. 35, 669 (1973); J. A. Hertz, Phys. Rev. B 14, 1165 (1976); A. J. Millis, Phys. Rev. B 48, 7183 (1993).

17 D. L. Maslov, arXiv:cond-mat/0506035 (Lecture notes for the LXXXI Les Houches Summer School "Nanoscopic Quantum Transport", 2004).

18 J. M. Luttinger and J. C. Ward, Phys. Rev. 118, 1417 (1960); G. Baym and L. P. Kadanoff, Phys. Rev. 124, 287 (1961).

19 P. Coleman, I. Paul, and J. Rech, Phys. Rev. B 72, 094430 (2005).

20 J. Kroha and P. Wolfle, J. Phys. Soc. Jpn. 74, 16 (2005); J. Kroha and P. Wolfle, Theoretical Methods for Strongly Correlated Electrons, D. Senechal, A.-M. Tremblay, and C. Bourbonnais Eds., CRM Series in Mathematical Physics (Springer, New York, 2003).

21 J. R. Schrieffer, Theory of Superconductivity (Westview Press, Colorado, 1999). 